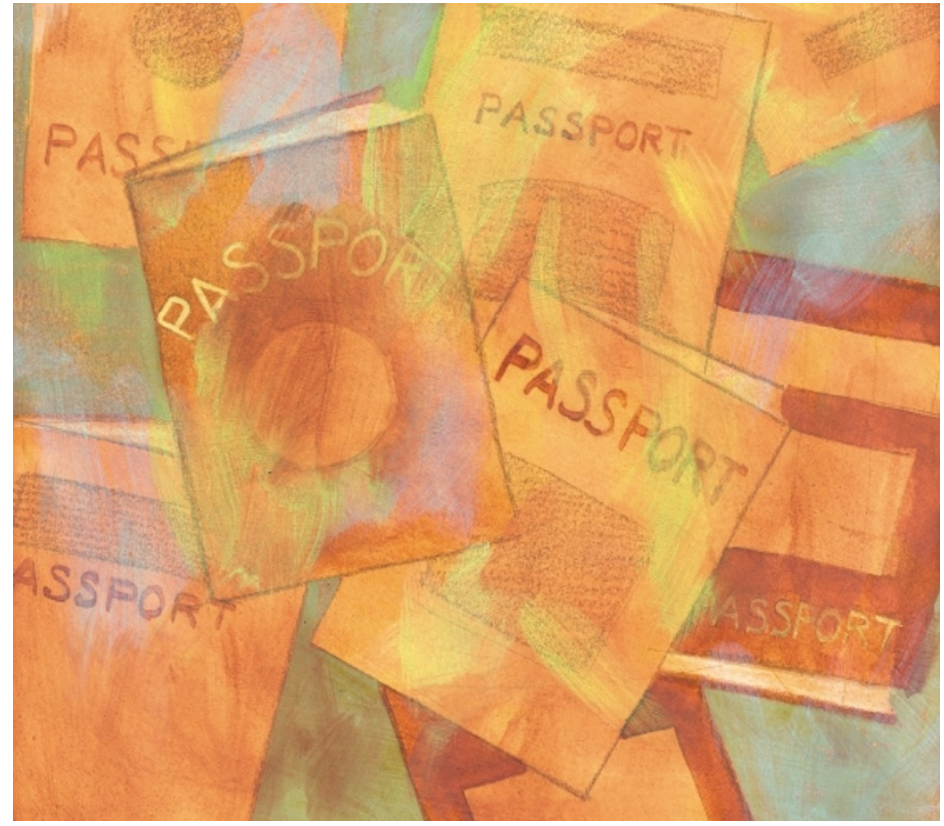

TELOMERES

\section{Controlled access}

Telomerase is responsible for adding telomeric DNA repeats to the ends of chromosomes. However, not all cells that produce catalytically active telomerase are capable of telomerase-dependent telomere maintenance. This suggested to Kathy Collins and colleagues that telomerase might be regulated by access to its substrate, as they now report in Nature Cell Biology.

The ribonucleoprotein (RNP) telomerase is a complex of protein and RNA that uses an integral RNA component as the template for telomere DNA synthesis by the inbuilt catalytic subunit, telomerase reverse transcriptase (TERT). Regulation occurs at the levels of both RNP accumulation and catalytic activation by RNP association with TERT. To investigate the possibility of a third level of regulation, the authors examined the subcellular localization of a green fluorescent protein-human TERT (GFP-hTERT) fusion protein by confocal microscopy. Conditions were chosen so that hTERT production limited telomerase activation, to ensure maximum incorporation of GFP-hTERT into telomerase RNP complexes.

In fibroblast cells, GFP-hTERT generally showed preferential nucleolar association, although some cells showed a more diffuse distribution. When cells were synchronized, Collins and co-workers found that most cells in G1 phase showed prominent nucleolar fluorescence. By contrast, cells in late S/G2 phase had a more diffuse intranuclear telomerase distribution, coincident with the timing of telomere replication.

They then went on to study the impact of oncogenic transformation and DNA damage on the subnuclear localization of catalytically active telomerase. In tumour and transformed cells, telomerase was almost completely dissociated from nucleoli at all stages of the cell cycle. And introduction of the SV40 genome with the T-antigen oncogene into a primary cell line was enough to release telomerase from nucleoli into the nucleoplasm.

Exposure of both primary and transformed cells to ionizing radiation caused a pronounced relocalization of GFP-hTERT to the nucleoli. This suggests that removing telomerase from damaged DNA reduces the potential for inappropriate repair of DNA breaks by telomere synthesis. The obvious next step will be to study the molecular mechanisms that underlie this phenomenon of subnuclear telomerase shuttling.

Arianne Heinrichs

\section{(2) References and links} al. Subnuclear shuttling of human telomerase induced by transformation and DNA damage.

FURTHER READING Blasco, M. Telomerase beyond telomeres. Nature Rev. Cancer $\mathbf{2}$, 627-633 (2002)

WEB SITE

Encyclopedia of Life Sciences:

http://www.els.net

Telomeres ORIGINAL RESEARCH PAPER Wong, J. M. Y. et Nature Cell Biol. 4,731-736 (2002)

\section{IN BRIEF}

\section{SIGNAL TRANSDUCTION}

Impaired B and T cell antigen receptor signaling in p110 $\delta$ PI 3-kinase mutant mice.

\section{Okkenhaug, K. et al. Science 297, 1031-1034 (2002)}

Class-IA phosphatidylinositol 3-kinases are composed of p85-p110 heterodimeric lipid kinases, and mammals express three isoforms of the catalytic p1 10 subunits. Researchers now show that mice expressing a catalytically inactive form of isoform $\mathrm{p} 110 \delta$ are impaired in antigen-receptor signalling in both B and T cells, and that the immune response is weakened. This suggests that the other two isoforms are not crucial for antigen-receptor signalling.

\section{CYTOSKELETON}

A role for regulated binding of $\mathrm{p} 150^{\text {G/ued }}$ to microtubule plus ends in organelle transport.

Vaughan, P. S. et al. J. Cell Biol. 158, 305-319 (2002)

Microtubule motor proteins are important for transport of cargo in the cell. In this report, Vaughan et al. studied the p $150^{\text {Glued }}$ subunit of the microtubule-associated protein dynactin using livecell imaging of green fluorescent protein (GFP)-p150 ${ }^{\text {Glued }}$ fusions. They showed that dynactin interacts dynamically with growing microtubule plus ends, and that $\mathrm{p} 150^{\text {Glued }}$ phosphorylation regulates this interaction. Analysis of GFP-p $150^{\text {Glued }}$ together with motile Golgi membranes indicates that the dynamic binding of dynactin to microtubules plus ends is important in the early stages of membrane transport.

\section{TECHNIQUE}

\section{Visualization of maltose uptake in living yeast cells by} fluorescent nanosensors.

Fehr, M. et al. Proc. Natl Acad. Sci. USA 99, 9846-9851 (2002)

To study the compartmentation and transport of metabolites, sensitive but noninvasive in vivo monitoring techniques are needed. Fehr et al. now report such a technique based on fluorescent nanosensors. Periplasmic binding proteins are suitable candidates for this protein-based nanosensor, and maltose-binding protein was chosen as a prototype. The binding of solute substrate to maltose-binding protein results in increased fluorescence that can be measured in a concentration-dependent manner.

\section{CELLULAR MICROBIOLOGY}

Helicobacter pylori SabA adhesin in persistent infection and chronic inflammation.

Mahdavi, J. et al. Science 297, 573-578 (2002)

Helicobacter pylori bacteria adhere to the gastric surface epithelium using adhesins that interact with specific host-cell receptors. Mahdavi and colleagues now identify sialyl-dimeric-Lewis- $\mathrm{X}$ as a new adherence receptor, and SabA as its corresponding bacterial adhesin. They also show that persistent infection upregulates the expression of sialyl-Lewis-X antigens, which could explain the extraordinary persistence of $H$. pylori infection. 